\title{
The Practice of OTC Dispensing by Community Pharmacist in Nepal
}

\author{
Sangita Shakya ${ }^{1}$, Susmita Sedai ${ }^{2}$ and Ronas Shakya ${ }^{3}$ \\ ${ }^{1}$ Assistant Professor, NIST, Kathmandu Nepal \\ ${ }^{2}$ Graduate Student, NIST, Kathmandu Nepal \\ ${ }^{3}$ Pharmacist, Patan Hospital, Kathmandu, Nepal
}

Corresponding Author

Sangita Shakya

Email: shakyasangita2020@gmail.com

\begin{abstract}
Over the counter (OTC) medications are the group of drugs that can be sold without the prescription of a registered medical practitioner to the consumers. Pharmacists are the most accessible health care professionals to the patient for the treatment of their minor illness. To provide appropriate advice to the patient at the time of dispensing and over-the-counter (OTC) medication counselling, community pharmacists need access to current and reliable information about medicines. A descriptive study was conducted on fifty pharmacists present in the community pharmacy of Kathmandu and Lalitpur districts. Predesigned validated questionnaire consisting of 16 questions were taken for the collection of data. Qualification of pharmacists, numbers of consumer asking for OTC medication in a week, most commonly dispensed OTC medicines by pharmacists, common symptoms told by consumers and average working hour of community pharmacists were recorded. The approval from the concerned authorities was taken. The study was a community based descriptive study done for 3 months. The data of the study was analyzed by using SPSS version 18. Out of 50 Pharmacists, 27 were males and 23 were females. Most of the study populations were qualified as assistant pharmacy. $82 \%$ of Community Pharmacy encountered more than ten consumers in a week. Analgesics and antipyretics, drugs for gastritis, antiprotozoals and antihistamines were the most commonly dispensed drugs by the pharmacies. The most common symptoms enquired by patients/consumers were fever (92\%), common cold $(90 \%)$ and headache $(86 \%)$ as reported by pharmacists respectively. Average working hours of community pharmacists were 67 hours per week. Few pharmacists knew the meaning of terms related to evidence-based health. Most respondents agreed that pharmacists have the necessary training to prescribe. There is increasing use of OTC medicines in Nepal. The study on community pharmacy practices regarding over the counter medicines can assist in evaluating pattern of use of OTC medicines by consumers thus help to reduce abuse and misuse of such medicines.
\end{abstract}

\section{KEYWORDS}

Community pharmacists, Community pharmacy, Over the Counter Medicine (OTC), Practices 


\section{INRODUCTION}

Over the counter (OTC) medicines are the group of drugs that can be sold without the prescription of a registered medical practitioner to the consumers. They are also known as nonprescription medicines (Ravichandran A, 2016 Nov;) According to the World Health Organization (WHO), OTC medicines are drugs approved by health authorities to treat minor ailments and symptoms. OTC medicines include Analgesics and Antipyretics, Anti-histaminics, Anti-diarrhoeals, Vitamins, Calcium, Anti-gastritic, Anti cold and laxatives.OTC drugs are considered an important component of primary health care system (Halila, 2015). If used appropriately, it can save the customers time and money by providing them safe and effective treatments for many commonly occurring illness (Maharjan, 2011). But inappropriate use of OTCs may lead to chances of adverse effect, drug interaction, drug abuse, misuse and antibiotic resistance (Halila, 2015)

The main objective of this study is to assess the pharmacists practice toward dispensing over the counter medicines in Community Pharmacy. Finally, proper use of OTC medications can improve the status of health care system. Pharmacists can play major role in proper use of OTC medication by giving information about instruction and possible side effect of drugs.

Nepal is developing countries and majority of people located in rural areas, where health care facilities are in scarce. In this situation, Patients can buy over the counter medicines for the treatment of their self diagnosed illness. Hence, this could increase the chances of abuse or misuse of OTC medicines (Sharma, 2017).

Proper use of OTC medications can improve the status of health care system. Like other developing countries, the use of OTC drugs is also increasing in Nepal. However, in Nepal, there is no adequate study regarding OTC medications had been done in Community Pharmacy.

By knowing the pattern of sales and dispensing of OTC drugs by pharmacists, this questionnaire based study helps to assess the practice of pharmacists toward dispensing over the counter medications.

\section{OBJECTIVES}

\section{General objective}

1. To study the community pharmacy practice regarding OTC medicines.

\section{Specific objectives}

1. To find out the qualification of pharmacists working in community pharmacy.

2. To find out the average numbers of consumers who ask for OTC medicines in a week.

3. To identify the commonly dispensed OTC medicines by pharmacists.

4. To assess the common symptoms for which the OTC medicines were dispensed.

5. To find out the average working hours of community pharmacists. 


\section{METHODOLOGY}

This was descriptive and prospective study conducted among fifty pharmacists and assistant pharmacists present in community pharmacy. The study was conducted at community pharmacy located in the Kathmandu and Lalitpur districts of Nepal which were the major districts of Nepal with high populations. The study was carried out from August to October 2018.

Predesigned validated questionnaire consisting of 16 questions were taken for the collection of data. A brief description of the study purpose was given to pharmacists before the questionnaire was distributed. After obtaining verbal consent, each pharmacist was provided a questionnaire regarding over the counter drugs. The community pharmacists were requested to complete the questionnaire. The obtained data was taken for analysis. Data entry, data checking, compiling and editing were done manually and data analysis was done as per the objectives of the study. Necessary editing was done immediately after data collection. Data analysis was done in SPSS version 18.

The approval from the concerned authorities and the Department of Pharmacy was taken before conducting the study. Verbal consent was taken from the community pharmacists. The data was collected after explaining the purpose of the study to the community pharmacists.

\section{RESULTS}

A total number of 110 community pharmacies were visited from the Kathmandu and Lalitpur districts of Nepal. Among them, Pharmacists were present in 66 (Kathmandu 34 and Lalitpur 32) Community pharmacies but only 50 Pharmacists (Kathmandu 25 and Lalitpur 25) were willing to participate in the study. Thus, average mean response of pharmacists was found to be $75.75 \%$. From this result, we can say that about $40 \%$ of community pharmacies were conducted by nonprofessional pharmacists or by the person who had taken orientation training (Figure 1). 


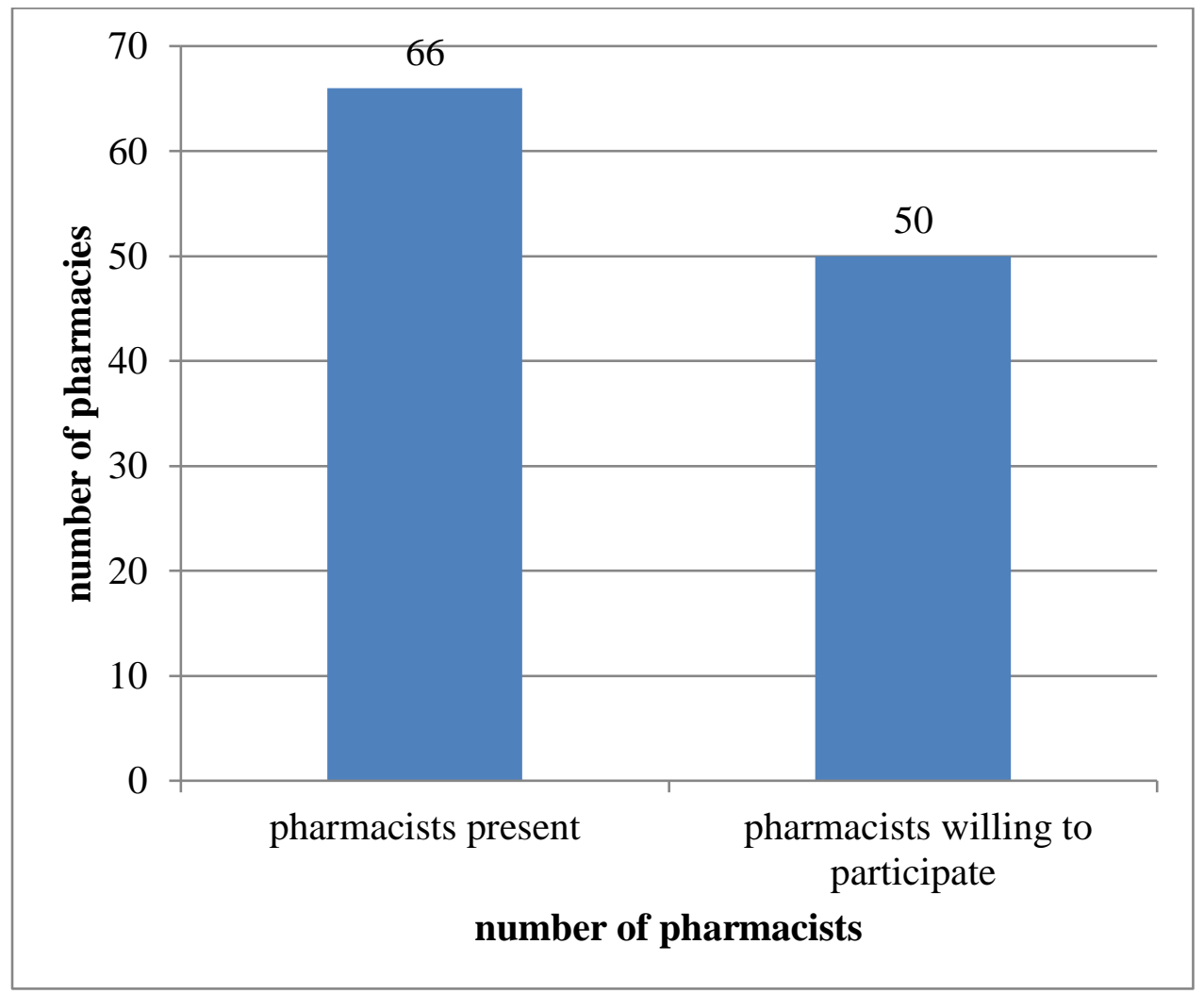

Figure1: Number of Pharmacy in Kathmandu and Lalitpur district

\section{Gender Distribution of Pharmacists}

A total of 50 pharmacists were included in this study, among them, 27(54\%) were male pharmacists and 23(46\%) were female Pharmacists. Our study showed that the numbers of male pharmacists were more than female pharmacists in community pharmacy of Kathmandu and Lalitpur districts of Nepal.

\section{Qualification of Pharmacists}

Figure 2 shows the qualification of pharmacist working in community pharmacies. Of the total pharmacists, $41(82 \%)$ pharmacists were qualified in Diploma in Pharmacy, $8(16 \%)$ having bachelor in Pharmacy and 1 (2\%) holding Master degree in Pharmacy. This result is similar to the study done in Indore city (2011), in which 38 (29.9\%) were qualified in Diploma in pharmacy, 18(14.2\%) having graduation in pharmacy and 5(3.9\%) holding Post-graduate (Master) in pharmacy.

From this result, we can reveal that majority of the pharmacists holding Diploma in pharmacy were present in the community pharmacies. 


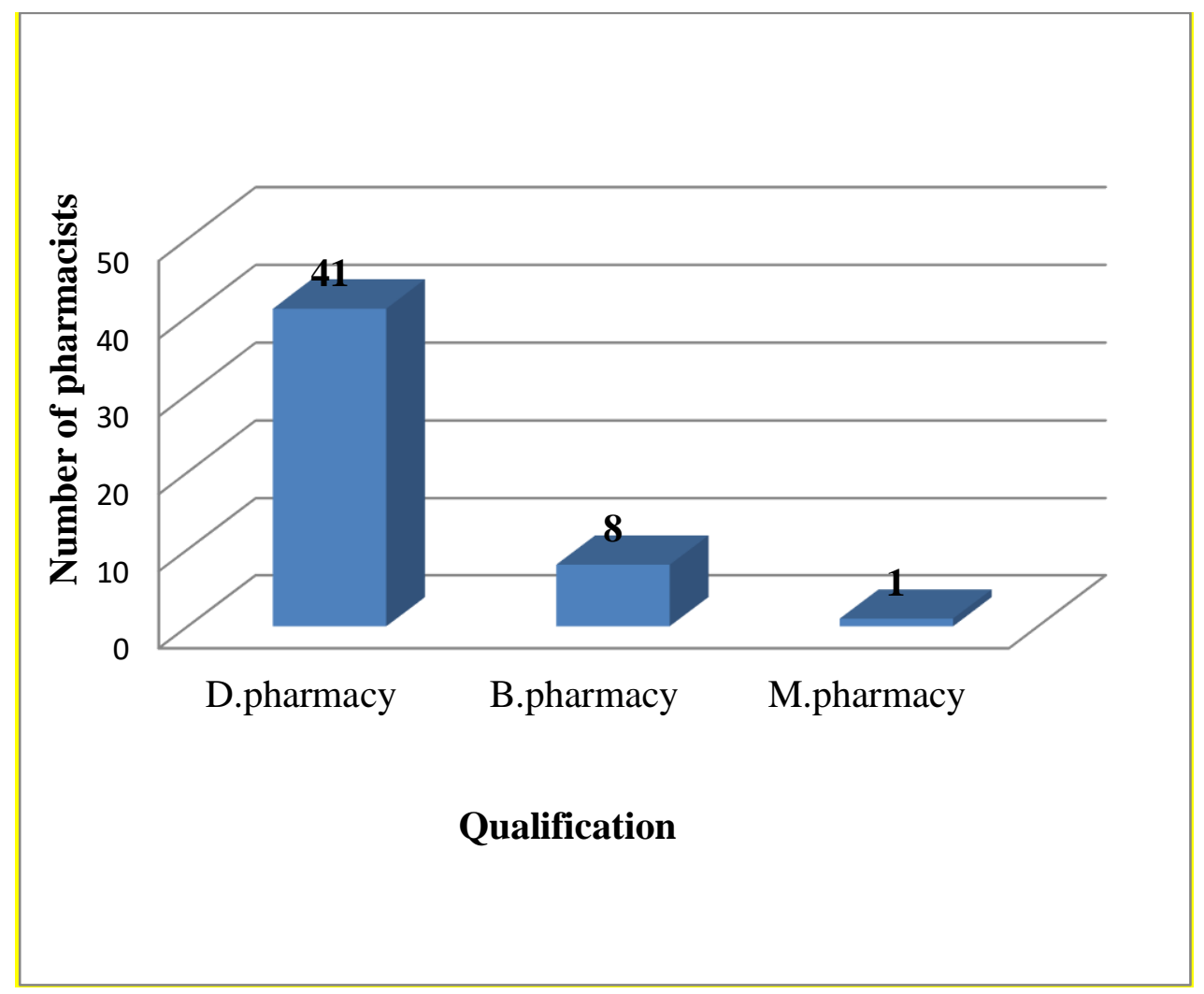

Figure 2: Qualification of Pharmacists Working in Community Pharmacy

\section{Commonly Dispensed OTC Medicines by the Pharmacies}

Analgesics and antipyretics (98\%), drugs for gastritis (96\%), antiprotozoals (80\%) and antihistamines (72\%) were the most commonly dispensed drugs by the pharmacies (Figure 3 ).

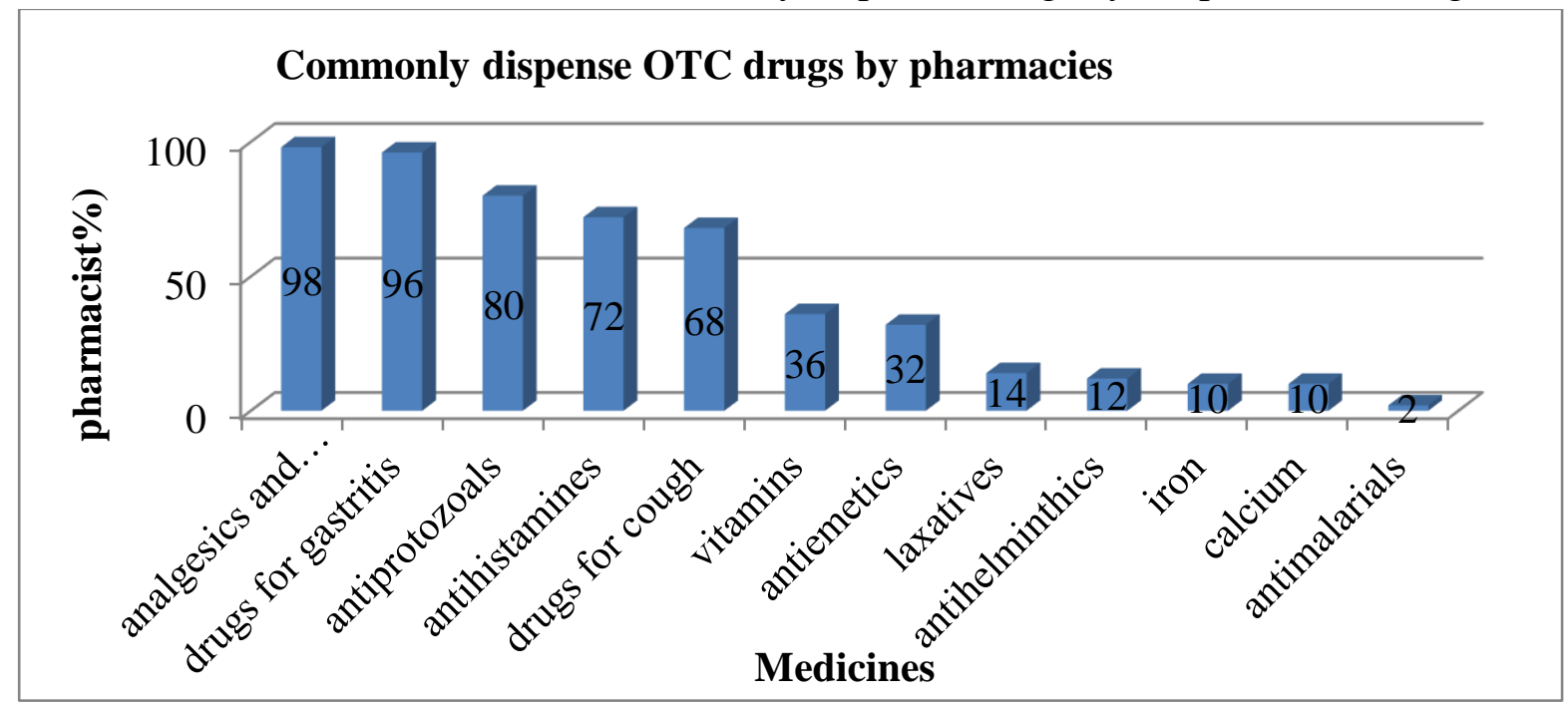

Figure 3: Commonly Dispensed OTC Medicines by the Pharmacies

Vol. 8. No. I 
ISSN: 2362-1303 (Paper) | eISSN: 2362-1311 (Online)

JOURNAL OF ADVANCED ACADEMIC RESEARCH (JAAR)

June 2021

\section{Pharmacists Practice and Opinion Regarding OTC Drugs}

According to the choice of OTC medications, $42 \%$ of pharmacists had dispensed by their own choice, $34 \%$ of pharmacist had dispensed OTC medications by consumer's choice, and $24 \%$ of pharmacists had dispensed OTC medications by both. Hence, Pharmacist choice predominated other factors.

Table 1: Pharmacists Practice and Opinion Regarding OTC Drugs

\begin{tabular}{lllc}
\hline & Questions & Reply & Response (\%) \\
\hline 1. & Choice of OTC medication & Consumer & 34 \\
& dispensed is made by & Pharmacist & 42 \\
& & Both & 24 \\
\hline $2 . \quad$ Are consumer complaints & Yes & 98 \\
& asked before dispensing OTC drugs? & No & 2 \\
\hline $3 . \quad$ Do you counsel the consumer & Yes [Always] & 26 \\
\hline before dispensing the drugs? & Yes [Sometimes] & 84 \\
& Do the consumers purchase & Brand name & 16 \\
& the drug by brand & Generic name & \\
\hline
\end{tabular}

OTC=Over-the-counter

\section{DISCUSSION}

A total number of 110 community pharmacies were visited from the Kathmandu and Lalitpur districts of Nepal. From the result, we can say that about $40 \%$ of community pharmacies were conducted by non professional pharmacists or by the person who had taken orientation training.

There was no pharmacy where less than 5 consumers come to ask for OTC medicines in a week. This result indicates that the prevalence of OTC medicines use is also high in Nepal. This result was similar to the study conducted by Ravichandran et al., (2016) in the Kolar district of India, where majority of pharmacies encountered more than 10 consumers asking for OTC medicines in a week (Ravichandran, 2016).

The analgesic and antipyretic were dispensed by majority of pharmacists; the reason could be due to the pain and fever were the most common symptoms encountered by the patients/consumers. In our study, it is inferred that $76 \%$ of community pharmacies have one pharmacist in one shift. Similarly, $12 \%$ of pharmacies have 3 pharmacists in one shift followed by $10 \%$ of pharmacies had two pharmacists and $2 \%$ of pharmacy has four pharmacists in one shift.

In this study, it was found that all of the pharmacists had dispensed OTC medications. However, only 20 pharmacists could define OTC drugs correctly without prior explanation. Among them, $75 \%$ of Pharmacists holding B. Pharmacy degree was able to define OTC correctly whereas only $29.26 \%$ of D. Pharmacy holders could define OTC correctly. Among 41 D.

Vol. 8. No. I

www.phdcentre.edu.np 
Pharmacy holders, $18 \%$ of them had no knowledge regarding OTC medicines. Similarly, our study complies with the study done in the Kolar district of India where $69 \%$ of pharmacists with B. Pharmacy degree holders and $31 \%$ of D. Pharmacy holders were able to define OTC correctly (Shankar, 2002).

Around $76 \%$ of pharmacists choose OTC brand based on consumer choice. $26 \%$ of pharmacists choose the brand based on the most commonly prescribed by the doctor in their locality. Nearly $14 \%$ of pharmacists choose the brand name based on cost of the drugs. And $2 \%$ relied their decision on medical representatives. Since the consumer choice is higher in selection of OTC brand, there are chances for adverse drug reactions or drug interactions with selfmedication because the consumers are unaware of the clinical situation or other medications (Foroughinia, 2016).

The present study found that $62 \%$ of the pharmacists said that dispensing OTC drugs are safe, but one should consult the physician first. According to $38 \%$ of pharmacists, OTC medications are safe to dispense. No pharmacist said OTC medicines are unsafe to dispense. From this study, we found that pharmacist's still feel dispensing OTC medicines without consulting physician is completely safe. In this scenario, it is the pharmacist who plays a prime role in educating and guiding the consumers to make informed decisions as he/she is the most accessible health-care professional to the consumers (Manju, 2016).

In similar study done in Indore city, consumer choice $(73.2 \%)$ was found to be higher than Pharmacist choice (24.8\%, Shroti, 2011).In our study, 98\% of the pharmacists were found to ask about consumer complaints before dispensing OTC medications.

A total of $74 \%$ of pharmacists always counsel and $26 \%$ pharmacists sometimes counsel the patient before dispensing OTC medication regarding the use of OTC medicines. By asking the consumer complaints, Pharmacists can understand the seriousness of the problem. This ultimately helps pharmacists in proper counselling of patients about their medications and occurring problem.

Most of the consumer purchased OTC drugs by brand name as reported by $84 \%$ of pharmacists which is greater than the study conducted by Manohar et al (2015), which showed that $53 \%$ of pharmacists were dispensed OTC drugs by brand name (Manohar, 2015). So this practice does not comply with the guideline given by WHO which states that every medicine should be dispensed by generic name (Sansgiry, 2017). 


\section{CONCLUSIONS}

All the pharmacists were qualified to dispense medication, but only $40 \%$ knew about OTC drugs. Majority of the pharmacy encountered more than 10 consumers in a week. Analgesics and antipyretics, drugs for gastritis, antiprotozoals and antihistamines were the most commonly dispensed OTC drugs by the pharmacists. Paracetamol, pantoprazole, combination of metronidazole and diloxanide furoate, cetrizine, vitamin B-complex, domperidone, albendazole and antitussives were the most commonly used OTC medicines in our community pharmacy. Fever, common cold and headache were the most common symptoms told by the patients/consumers. Average working hour of pharmacists was found to be 67 hours per week.

\section{RECOMMENDATION}

Pharmacists have to remain up-to-date in their knowledge; to make such practice is safe and useful. Thus to reduce abuse and misuse and to monitor dispensing practice of OTC medicines, the Government of Nepal and the concerned authority should adopt strategies and take necessary action.

\section{LIMITATION}

This research was conducted within limitation of time and resources. So the study could not be generalized. The study was limited to small sample size in the Kathmandu and Lalitpur district of Nepal. Pharmacist intervention was not conducted in this study.

Received: 25 May 2021

Accepted: 23 June 2021

Published: 28 June 2021

\section{REFERENCES}

Foroughinia, F., Zarei, P. (2016). Evaluation of knowledge, attitude, and practice of community pharmacists toward administration of over-the-counter drugs for the treatment of diarrhea in children: A pretest-posttest survey. Journal of Research in Pharmacy Practice, 5(3), 200-4.

Halila, G.C., Junior, E.H. (2015). The practice of OTC counseling by community pharmacists in Parana, Brazil. Pharmacy Practice (Granada), 13(4), 597.

Maharjan, M., Adhikari, K. (2011). Practices on over the counter medication among mothers of under five aged children attending child OPD at friends of Shanta Bhawan, Boudha, Kathmandu. Health prospect, 10, 19-21

Manju, C.S., Pichan, I., Jemsheena, P.M. ( 2016). Role of community pharmacist in self medication. Pharmaceutical and Biological Evaluations, 3(6), 541-546.

Manohar, H.D., Manohar, H.L. (2015). Impact of knowledge and attitude on practices of over the counter medications. IEOM Society. 
ISSN: 2362-1303 (Paper) | eISSN: 2362-1311 (Online)

Ravichandran, A., Basavareddy, A. (2016). Perception of pharmacists regarding over-the-counter medication: A survey. Indian Journal of Pharmacology, 48(6), 729-732.

Sansgiry, S.S., Bhansali, A.H., Bapat, S.S., Xu, Q. (2017). Abuse of over-the-counter medicines: a pharmacist's perspective. Integrated PharmacyRresearch and Practice.

Shankar, P.R., Partha, P, Shenoy, N. (2002). Self-medication and non-doctor prescription practices in Pokhara valley, Western Nepal: a questionnaire-based study. BMC Family Practice, 3(1), 17

Sharma, D., Gurung, D., Kafle, R., Singh, S. (2017). Knowledge and practice on over-thecounter drugs among adults of age group 20 and above residing in Chapapani-12, Pokhara, Kaski, Nepal. International Journal of Scientific Reports, 3(3), 79-86

Shroti, R., Nayak, N., Rajput, M.S. (2011). Astudy on over the counter drugs in retail pharmacies in Indore city. Der Pharmacia Lettre, 3(3),133-138. 